\title{
ON THE GROWTH OF THE SPECTRAL MEASURE
}

\author{
A. BOUMENIR \\ King Fahd University of Petroleum and Minerals \\ Department of Mathematical Sciences \\ Dhahran 31262, SAUDI ARABIA
}

(Received May 26, 1992 and in revised form June 12, 1996)

\begin{abstract}
We are concerned with the asymptotics of the spectral measure associated with a self-adjoint operator. By using comparison techniques we shall show that the eigenfunctionals of $L_{2}$ are close to the eigenfunctionals $L_{1}$ if and only if $d \Gamma_{1} \asymp d \Gamma_{2}$ as $\lambda \rightarrow \infty$.

KEY WORDS AND PHRASES: Spectral asymptotics, spectral function, Sturm-Liouville operators.
\end{abstract}

1991 AMS SUBJECT CLASSIFICATION CODES: 34L20.

\section{INTRODUCTION}

We would like to obtain a relation between the growth of the spectral measure of a self-adjoint operator and the behaviour of its eigenfunctionals. In this study we shall assume that we have two "close" self-adjoint operators acting in the same separable Hilbert space, $H$ say. Without loss of generality we can assume that both operators have simple spectra. To this end, let us denote by $\varphi(\lambda)$ and $y(\lambda)$ the eigenfunctionals of $L_{1}$ and $L_{2}$ respectively. Recall that the spectrum of a self-adjoint operator is defined by

$$
\forall \lambda \in \sigma_{\imath} \quad \exists \varphi_{\imath, n} \in D_{\left\{L_{1}\right\}} /\left\|\varphi_{\imath, n}\right\|=1 \text { and }\left\|L_{2} \varphi_{\imath, n}-\lambda \varphi_{\imath, n}\right\| \stackrel{n \rightarrow \infty}{\longrightarrow} 0
$$

where $i=1,2$. In case $\lambda$ is in the continuous spectrum the sequence is not compact in the Hilbert space $H$. For this we can assume the existence of a countably normed perfect space $\Phi$, such that

$$
\Phi \hookrightarrow H \hookrightarrow \Phi^{\prime}
$$

where the embeddings are compact, for further details see [1] and [2]. For the sake of simplicity we shall assume that the embeddings are given by the identities and so

$$
f \in \Phi \quad \psi \in H \quad(f, \psi) \equiv<f, \psi>_{\Phi \times \Phi^{\prime}} .
$$

Since the sequence $\varphi_{n}$ is bounded in $H$, it is then compact in $\Phi^{\prime}$, which implies

$$
\varphi_{n} \stackrel{\Phi^{\prime}}{\rightarrow} \varphi(\lambda) \in \Phi^{\prime}
$$

and similarly for the operator $L_{2}$; Since both operators are acting in the same Hilbert space $H$, we shall assume that the space $\Phi^{\prime}$ contains both systems of eigenfunctionals; i.e.,

$$
\{y(\lambda)\} \subset \Phi^{\prime} \text { and }\{\varphi(\lambda)\} \subset \Phi^{\prime} .
$$

Recall that the system $\{y(\lambda)\}$ helps define an isometry for $L_{2}$

Similarly for $\varphi(\lambda)$;

$$
\begin{gathered}
\forall f \in \Phi \quad f \longrightarrow \hat{f}^{2}(\lambda) \equiv<f, y(\lambda)>_{\Phi \times \Phi^{\prime}} \\
f=\int \overline{\hat{f}^{2}(\lambda)} y(\lambda) d \Gamma_{2}(\lambda) \quad \text { where } \quad \hat{f}^{2}(\lambda) \in L_{d \Gamma_{2}(\lambda)}^{2}
\end{gathered}
$$




$$
\begin{aligned}
& \forall f \in \Phi \quad f \longrightarrow \hat{f}^{1}(\lambda) \equiv<f, \varphi(\lambda)>_{\Phi \times \Phi^{\prime}}, \\
& f=\int \overline{\hat{f}^{1}(\lambda)} \varphi(\lambda) d \Gamma_{1}(\lambda) \text { where } \hat{f}^{1}(\lambda) \in L_{d \Gamma_{1}(\lambda)}^{2}
\end{aligned}
$$

These transforms define isometries, and Parseval equality yields

$$
\int_{\sigma_{1}} \hat{f}^{1}(\lambda) \overline{\hat{\psi}^{1}(\lambda)} d \Gamma_{1}(\lambda)=(f, \psi)_{H}=\int_{\sigma_{2}} \hat{f}^{2}(\lambda) \overline{\hat{\psi}^{2}(\lambda)} d \Gamma_{2}(\lambda) .
$$

where the nondecreasing functions $\Gamma_{1}(\lambda)$ and $\Gamma_{2}(\lambda)$ are called the spectral measures associated with $L_{1}$ and $L_{2}$, respectively. It is these functions that we would like to estimate as $\lambda \rightarrow \infty$.

In all that follows $y(\lambda) \sim \varphi(\lambda)$ as $\lambda \rightarrow \infty$ means $\forall f \in \Phi$,

$$
\hat{f}^{1}(\lambda) \asymp \hat{f}^{2}(\lambda) \text { as } \lambda \rightarrow \infty
$$

and $d \Gamma_{1}(\lambda) \sim d \Gamma_{2}(\lambda)$ as $\lambda \rightarrow \infty$ means that $\forall F \in L_{d \Gamma_{1}(\lambda)}^{1} \cap L_{d \Gamma_{2}(\lambda)}^{1}$

$$
\int_{\lambda}^{\infty} F(\eta) d \Gamma_{1}(\eta) \asymp \int_{\lambda}^{\infty} F(\eta) d \Gamma_{2}(\eta) \quad \text { as } \quad \lambda \rightarrow \infty
$$

In this work, we shall try to answer the following problem:

Statement of the Problem: under what conditions

$$
y(\lambda) \sim \varphi(\lambda) \text { as } \lambda \rightarrow \infty \Leftrightarrow d \Gamma_{1}(\lambda) \sim d \Gamma_{2}(\lambda) \text { as } \lambda \rightarrow \infty .
$$

In order to answer the above question, we shall compare the self-adjoint operators $L_{1}$ and $L_{2}$, see [3]. Recall that a shift operator or transmutation is defined by

$$
y(\lambda)=V \varphi(\lambda) \quad \lambda \in \sigma_{1}
$$

Clearly the definition of $V$ depends on $\sigma_{2}$ and $\sigma_{1}$ and we shall agree to set

$$
\begin{gathered}
y(\lambda)=0 \text { if } \lambda \notin \sigma_{2}, \text { and } \varphi(\lambda)=0 \text { if } \lambda \notin \sigma_{1} \\
y(\lambda)=V \varphi(\lambda) \quad \lambda \in \sigma_{2} \subset \sigma_{1} \subset R .
\end{gathered}
$$

Condition $\sigma_{2} \subset \sigma_{1}$ insures that $V 0=0$ and so defines an operator on the algebraic span of $\{\varphi(\lambda)\}$. Thus it is clear that in order for $V$ and $V^{-1}$ to exist as linear operator it is necessary that $\sigma_{2} \subset \sigma_{1}$ and $\sigma_{1} \subset \sigma_{2}$

$$
\sigma_{2} \equiv \sigma_{1}
$$

It is readily seen that $\{\varphi(\lambda)\}$ form a complete set in the reflexive space (perfect) $\Phi^{\prime}$, and so the space generated by $\{\varphi(\lambda)\}$ is dense in $\Phi^{\prime}$. Consequently $V$ is densely defined. This in turns allows us to define the adjoint operator $V^{\prime}: \Phi \rightarrow \Phi$.

\section{MAIN RESULTS}

We shall agree to say $\Gamma_{1}(\lambda)$ is Abs- $d \Gamma_{2}$ if there exists $g(\eta) \in L_{d \Gamma_{2}}^{1, l o c}$ such that

$$
\Gamma_{1}(\lambda)=\int_{0}^{\lambda} g(\eta) d \Gamma_{2}(\eta)+\Gamma_{1}(0)
$$

This fact shall be denoted by

$$
g(\lambda) \equiv \frac{d \Gamma_{1}}{d \Gamma_{2}}(\lambda) \in L_{d \Gamma_{2}}^{1, l o c}
$$

In this case the condition $d \Gamma_{1}(\lambda) \sim d \Gamma_{2}(\lambda)$ in the statement of the problem can be restated as $g(\lambda) \asymp 1$ as $\lambda \rightarrow \infty$. Recall that due to reflexivity of the space $\Phi$, the operator $V^{\prime}$ is defined in $\Phi$ and since $\Phi \hookrightarrow H, V^{\prime}$ is actually defined in $H$. Let us denote this extension to the space $H$ by $\tilde{V}$. Since we are interested in the case where $y(\lambda) \sim \varphi(\lambda)$ we can expect $V$ to be bounded. In this regard we have the following result: 
Theorem 1: If the extension $\tilde{V}: H \rightarrow H$, is a bounded operator then $\Gamma_{1}(\lambda)$ is $d \Gamma_{2}$-ABS continuous.

Proof: It is clear that for $f \in D_{V^{\prime}}$

$$
\begin{aligned}
<f, y(\lambda)>_{\boldsymbol{\Phi} \times \Phi^{\prime}} & =\langle f, V \varphi(\lambda)\rangle_{\boldsymbol{\Phi} \times \Phi^{\prime}} \\
& =\left\langle V^{\prime} f, \varphi(\lambda)\right\rangle_{\boldsymbol{\Phi} \times \Phi^{\prime}}
\end{aligned}
$$

In other words

$$
\hat{f}^{2}(\lambda)={\widehat{V^{\prime} f}}^{1}(\lambda) .
$$

Equation 2.1 obviously holds for $f \in H$. Indeed let $f_{n} \in D_{V^{\prime}} \subset H$ such that $f_{n} \stackrel{H}{\rightarrow} f \in H$. Given that $\tilde{V}$ is a bounded operator in $H$, we obviously have $\tilde{V} f_{n} \rightarrow \tilde{V} f$. Using the fact that $\forall n, \quad \hat{f}_{n}^{2}(\lambda)={\widehat{V} f_{n}}^{1}(\lambda)$ and the isometries are bounded operators we have $\hat{f}_{n}^{2} \rightarrow \hat{f}^{2}$ and ${\widehat{\bar{V} f_{n}}}^{1} \rightarrow$ $\widehat{\hat{V} f}$. Therefore

$$
\hat{f}^{2}(\lambda)=\widehat{\widehat{V} f}^{1}(\lambda) \quad f \in H .
$$

From which we deduce that $\forall f \in H$

$$
\begin{aligned}
\int \hat{f}^{2}(\lambda) \overline{\hat{f}^{2}(\lambda)} d \Gamma_{1}(\lambda) & =\int \widehat{\tilde{V}^{\prime} f} \overline{{\widehat{V^{\prime}}}^{1}} d \Gamma_{1}(\lambda) \\
& =\left(\tilde{V}^{\prime} f, \tilde{V}^{\prime} f\right) \\
& =\left\|\tilde{V}^{\prime} f\right\|^{2} \\
& \leq c\|f\|^{2} \\
& \leq c \int\left|\hat{f}^{2}(\lambda)\right|^{2} d \Gamma_{2}(\lambda) \quad \forall f \in H .
\end{aligned}
$$

Thus each $d \Gamma_{2}$ negligible set is a $d \Gamma_{1}$ negligible set. Henceforth $\Gamma_{1}(\lambda)$ to be $d \Gamma_{2}(\lambda)$-Abs continuous. The above inequality is exactly a sufficient condition for the Radon-Nikodym theorem to hold, see [4].

In all that follows we shall assume that $d \Gamma_{1}(\lambda)$ is $d \Gamma_{2}-A b s$ continuous which is denoted by

$$
g(\lambda) \equiv \frac{d \Gamma_{1}}{d \Gamma_{2}}(\lambda)
$$

We now need to define a function of an operator, namely $g\left(L_{2}\right)$ for the next result:

$$
\begin{array}{ll}
\Phi & \stackrel{g\left(L_{2}\right)}{\longrightarrow} H \\
f \longrightarrow & \longrightarrow\left(L_{2}\right) f \equiv \int g(\lambda) \overline{\hat{f}^{2}(\lambda)} y(\lambda) d \Gamma_{2}(\lambda) .
\end{array}
$$

Theorem 2: Assume that $V$ admits closure in $\Phi^{\prime}$ and $\Gamma_{1}$ is $A b s-d \Gamma_{2}(\lambda)$ then

$$
\forall \psi \in D_{V^{\prime}} \subset \Phi \quad\left(\sqrt{\frac{d \Gamma_{2}}{d \Gamma_{1}}\left(L_{2}\right)}\right)^{\prime}\left(\sqrt{\frac{d \Gamma_{2}}{d \Gamma_{1}}\left(L_{2}\right)}\right) \psi=\bar{V} V^{\prime} \psi \quad \text { in } \Phi^{\prime} .
$$

Proof: From equation 2.1 and the fact that the embeddings are defined by identities, we deduce that $\forall f, \psi \in D_{V^{\prime}} \subset \Phi$

$$
\begin{aligned}
\int \hat{f}^{2}(\lambda) \overline{\hat{\psi}^{2}(\lambda)} d \Gamma_{1}(\lambda) & =\int \widehat{V^{\prime} f} \overline{\widehat{V}^{\prime} \psi} d \Gamma_{1}(\lambda) \\
& =\left(V^{\prime} f, V^{\prime} \psi\right) \\
& =\left\langle V^{\prime} f, V^{\prime} \psi>_{\Phi \times \Phi^{\prime}}\right. \\
& =\left\langle f, \bar{V} V^{\prime} \psi>_{\Phi \times \Phi^{\prime}}\right.
\end{aligned}
$$


However the left handside of equation 2.3 can rewritten as

$$
\begin{aligned}
\int \hat{f}^{2}(\lambda) \overline{\hat{\psi}^{2}(\lambda)} d \Gamma_{1}(\lambda) & =\int \hat{f}^{2}(\lambda) \overline{\hat{\psi}^{2}(\lambda)} g(\lambda) d \Gamma_{2}(\lambda) \\
& =\int \sqrt{g(\lambda)} \hat{f}^{2}(\lambda) \overline{\sqrt{g(\lambda)} \hat{\psi}^{2}} d \Gamma_{2}(\lambda) \\
& =\int \sqrt{\sqrt{g\left(L_{2}\right)}} f^{2} \overline{\sqrt{g\left(L_{2}\right)} \psi^{2}} d \Gamma_{2}(\lambda) \\
& =\left(\sqrt{g\left(L_{2}\right)} f, \sqrt{g\left(L_{2}\right)} \psi\right) \\
& =\left\langle f, \sqrt{g\left(L_{2}\right)} \cdot \sqrt{g\left(L_{2}\right)} \psi\right\rangle_{\Phi \times \Phi^{\prime}}
\end{aligned}
$$

Observe that if we set $f=\psi$ in equations 2.4 and 2.5 then we would obtain

$$
\left\|\sqrt{g\left(L_{2}\right)} f\right\|=\left\|V^{\prime} f\right\|
$$

from which we deduce that $D_{V^{\prime}} \subset D_{\sqrt{g\left(L_{2}\right)}} \subset \Phi$, from we obtain

$$
\forall \psi \in D_{V^{\prime}} \quad \sqrt{g\left(L_{2}\right)} \sqrt{g\left(L_{2}\right)} \psi=\bar{V} V^{\prime} \psi
$$

Remark: Observe that both operators $\sqrt{g\left(L_{2}\right)} \sqrt{g\left(L_{2}\right)}$ and $\bar{V} V^{\prime}$ are mappings from $\Phi \longrightarrow \Phi^{\prime}$. It is easy to see that if we restrict equation 2.7 to

$$
f \in D_{g\left(L_{2}\right)} \equiv\left\{f \in \Phi / g(\lambda) \hat{f}^{2}(\lambda) \in L_{d \Gamma_{2}}^{2}\right\}
$$

then it reduces to

$$
\forall f \in D_{V^{\prime}} \cap D_{g\left(L_{2}\right)} \quad \frac{d \Gamma_{1}}{d \Gamma_{2}}\left(L_{2}\right)=g\left(L_{2}\right)=\bar{V} V^{\prime} \quad \text { in } \Phi^{\prime}
$$

The next result describes the domain of $\tilde{V}^{\prime}$.

Theorem 3: $\tilde{V}$ is densely defined if and only if $L_{d \Gamma_{1}(\lambda)}^{2} \cap L_{d \Gamma_{2}(\lambda)}^{2}$ is dense in $L_{d \Gamma_{2}(\lambda)}^{2}$.

Proof: From equation 2.2 it is readily seen that

$$
f \in D_{\bar{v}} \Leftrightarrow \hat{f}^{2}(\lambda) \in L_{d \Gamma_{1}(\lambda)}^{2} \cap L_{d \Gamma_{2}(\lambda)}^{2}
$$

Then use the fact that $f \longrightarrow \hat{f}^{2}$ is an isometry between $H$ and $L_{d \Gamma_{2}(\lambda)}^{2}$.

This work is based on the following result.

Theorem 4: Assume that

- $V$ admits closure in $\Phi^{\prime}$

- $\Gamma_{1}$ is Abs- $d \Gamma_{2}(\lambda)$

- $\bar{V}^{-1}$ exists

- $\bar{V}: \Phi \longrightarrow \Phi$ is a bounded operator

then

$$
g(\lambda) \varphi(\lambda)-y(\lambda)=\left(V^{\prime}-1\right) y(\lambda) \quad \text { in } \Phi^{\prime} .
$$

Proof: Notice that conditions of Theorem 2 hold and so it follows that

$$
\sqrt{g\left(L_{2}\right)} \cdot \sqrt{g\left(L_{2}\right)}=\bar{V} V^{\prime} \text { in } \Phi^{\prime} \text {. }
$$

By the above condition we have that $\sqrt{g\left(L_{2}\right)} \sqrt{g\left(L_{2}\right)} f \in \Phi$ if $f \in D_{V^{\prime}} \subset \Phi$. However since it is assumed that $\bar{V}^{-1}$ exists, then equation 2.8 yields

$$
\bar{V}^{-1}\left(\sqrt{g\left(L_{2}\right)}\right)^{\prime} \sqrt{g\left(L_{2}\right)}=V^{\prime} \text { in } \Phi^{\prime}
$$

In order to proceed further we need to extend the operator $V^{\prime}$ to $\Phi^{\prime}$. For this observe that since $\bar{V}: \Phi \longrightarrow \Phi$ is a bounded operator, $\overline{V^{\prime}}=V^{\prime}$ is a bounded operator in $\Phi^{\prime}$. Hence $V^{\prime}$ is defined for all elements in $\Phi^{\prime}$, and in particular for $y(\lambda)$, thus 


$$
V^{-1} \sqrt{g\left(L_{2}\right)} \cdot \sqrt{g\left(L_{2}\right)} y(\lambda)=V^{\prime} y(\lambda) .
$$

We now need to compute $\left.\sqrt{g\left(L_{2}\right)}\right)^{\prime} \sqrt{g\left(L_{2}\right)} y(\lambda)$. Let $f \in D_{V^{\prime}} \subset \Phi$ then

$$
\begin{aligned}
<f,{\sqrt{g\left(L_{2}\right)}}^{\prime} \sqrt{g\left(L_{2}\right)} y(\lambda)>_{\Phi \times \Phi^{\prime}} & =\left\langle\sqrt{g\left(L_{2}\right)} f, \sqrt{g\left(L_{2}\right)} y(\lambda)>_{\Phi \times \Phi^{\prime}}\right. \\
& =<\sqrt{g\left(L_{2}\right)} \sqrt{g\left(L_{2}\right)} f, y(\lambda)>_{\Phi \times \Phi^{\prime}} \\
& =\frac{\sqrt{g(\lambda)}}{g(\lambda)} \hat{f}^{2}(\lambda) \\
& =g(\lambda) \hat{f}^{2}(\lambda) \\
& =<f, g(\lambda) y(\lambda)>
\end{aligned}
$$

where we have used the fact that $\sqrt{g\left(L_{2}\right)} \sqrt{g\left(L_{2}\right)} f=\bar{V} V^{\prime} f \in \Phi$. Hence

$$
{\sqrt{g\left(L_{2}\right)}}^{\prime} \sqrt{g\left(L_{2}\right)} y(\lambda)=g(\lambda) y(\lambda) \text { in } \Phi^{\prime} d \Gamma_{2} \text { a.e. }
$$

where $g(\lambda) \equiv \frac{d \Gamma_{1}}{d \Gamma_{2}}(\lambda)$ is a real function. Hence we have

$$
g(\lambda) \bar{V}^{-1} y(\lambda)=V^{\prime} y(\lambda) .
$$

Since by definition we have $\bar{V}^{-1} y(\lambda)=\varphi(\lambda)$ we obtain

$$
g(\lambda) \varphi(\lambda)-y(\lambda)=\left(V^{\prime}-1\right) y(\lambda) \text { in } \Phi^{\prime} .
$$

We easily deduce the following result:

- Corollary 1: Let conditions of Theorem 4 hold then

$$
g(\lambda) \varphi(\lambda)-y(\lambda) \stackrel{\Phi^{\prime}}{\longrightarrow} 0 \Leftrightarrow\left(V^{\prime}-1\right) y(\lambda) \stackrel{\Phi^{\prime}}{\longrightarrow} 0
$$

Corollary 2: Let conditions of Theorem 4 hold and $\left(V^{\prime}-1\right) y(\lambda) \stackrel{\Phi^{\prime}}{\longrightarrow} 0 \quad \lambda \rightarrow \infty$ then

$$
g(\lambda) \sim 1 \text { as } \lambda \rightarrow \infty \Leftrightarrow \varphi(\lambda)-y(\lambda) \stackrel{\Phi^{\prime}}{\longrightarrow} 0 \text { as } \lambda \rightarrow \infty .
$$

Proof: By hypothesis and Corollary 1 we have $\forall f \in \Phi$

$$
g(\lambda) \hat{f}^{1}(\lambda)-\hat{f}^{2}(\lambda) \rightarrow 0 \text { as } \lambda \rightarrow \infty .
$$

Thus if $g(\lambda) \rightarrow 1$ then $\hat{f}^{1}(\lambda)-\hat{f}^{2}(\lambda) \rightarrow 0$ which means that $\varphi(\lambda)-y(\lambda) \stackrel{\Phi^{\prime}}{\longrightarrow} 0$ as $\lambda \rightarrow \infty$. Conversely $\hat{f}^{1}(\lambda)-\hat{f}^{2}(\lambda) \rightarrow 0$ together with $y(\lambda)-g(\lambda) \varphi(\lambda) \stackrel{\Phi^{\prime}}{\longrightarrow} 0$ implies that

$$
g(\lambda) \hat{f}^{1}(\lambda)-\hat{f}^{2}(\lambda) \rightarrow 0
$$

i.e. $g(\lambda) \rightarrow 1$ as $\lambda \rightarrow \infty$.

Corollary 2 suggests to write $V=1+K$. In this case Theorem 2 would read

$$
g(\lambda) \varphi(\lambda)-y(\lambda) \stackrel{\Phi^{\prime}}{\longrightarrow} 0 \Leftrightarrow K^{\prime} y(\lambda) \stackrel{\Phi^{\prime}}{\longrightarrow} 0 \text { as } \quad \lambda \rightarrow, \infty .
$$

The question we would like to answer now is under what condition would

$$
K^{\prime} y(\lambda) \stackrel{\Phi^{\prime}}{\longrightarrow} 0 \text { as } \lambda \rightarrow \infty .
$$

First we need to observe that the above convergence holds in $\Phi^{\prime}$. Indeed by construction the function $y(\lambda)$ is in $\Phi^{\prime}$ and so the operator $K^{\prime}$ originally was defined in $\Phi$ must be extended to $\Phi^{\prime}$. This is easily achieved if the operator $K$, i.e. $\bar{V}$, is bounded in $\Phi \longrightarrow \Phi$.

Theorem 5: Let

- $V: \Phi \longrightarrow \Phi$ be a bounded operator.

- $K \equiv \bar{V}-1$, be such that $\Phi \stackrel{L_{2} K}{\longrightarrow} H$ is densely defined in $\Phi$

then

$$
K^{\prime} y(\lambda) \stackrel{\Phi^{\prime}}{\longrightarrow} 0 \text { as } \lambda \rightarrow \infty .
$$

Proof: Recall that for each $\lambda$, there exists a bounded sequence $\varphi_{n, \lambda} \in D_{L_{2}}$ such that 


$$
\varphi_{n, \lambda} \in D_{L_{2}}, \quad\left\|\varphi_{n, \lambda}\right\|=1, \text { and }\left\|L_{2} \varphi_{n, \lambda}-\lambda \varphi_{n, \lambda}\right\| \longrightarrow 0
$$

The last condition can be written as

$$
\lambda \varphi_{n, \lambda}=L_{2} \varphi_{n, \lambda}+\epsilon(n, \lambda)
$$

where $\epsilon(n, \lambda) \rightarrow 0$ in $H$ as $n \rightarrow \infty$. This allows us to obtain the following limit

$$
\begin{aligned}
<f, K^{\prime} y(\lambda)>_{\boldsymbol{\Phi} \times \Phi^{\prime}} & =<K f, y(\lambda)>_{\boldsymbol{\Phi} \times \Phi^{\prime}} \\
& =\lim _{n \rightarrow \infty}<K f, \varphi_{n, \lambda}>_{\Phi \times \Phi^{\prime}} \\
& =\frac{1}{\lambda} \lim _{n \rightarrow \infty}\left(\lambda \varphi_{n, \lambda}, K f\right) \\
& =\frac{1}{\lambda} \lim _{n \rightarrow \infty}\left(L_{2} \varphi_{n, \lambda}+\epsilon(n, \lambda), K f\right) \\
& =\frac{1}{\lambda} \lim _{n \rightarrow \infty}\left(L_{2} \varphi_{n, \lambda}, K f\right)+\frac{1}{\lambda} \lim _{n \rightarrow \infty}(\epsilon(n, \lambda), K f) \\
& =\frac{1}{\lambda} \lim _{n \rightarrow \infty}\left(\varphi_{n, \lambda}, L_{2} K f\right)+\frac{1}{\lambda} \lim _{n \rightarrow \infty}(\epsilon(n, \lambda), K f) \\
& \leq \frac{1}{\lambda}\left\|\varphi_{n, \lambda}\right\|\left\|L_{2} K f\right\|+\frac{1}{\lambda} \lim _{n \rightarrow \infty} \|(\epsilon(n, \lambda)\|\| K f \|
\end{aligned}
$$

So as $\lambda \rightarrow \infty$ we shall obtain $\left\langle f, K^{\prime} y(\lambda)>_{\Phi \times \Phi^{\prime}} \longrightarrow 0\right.$. This last limit means that

$$
K^{\prime} y(\lambda) \stackrel{\Phi^{\prime}}{\longrightarrow} 0 \text { as } \lambda \rightarrow \infty .
$$

Recall that in order for the conclusion to hold we need $L_{2} K$ to be at least densely defined in $\Phi$.

Remark: The condition $V: \Phi \longrightarrow \Phi$ bounded can be replaced by densely defined. This forces us to use Baire's Theorem to obtain the density of $\Phi \cap D_{V} \cap D_{L_{2} K}$ in $\Phi$.

Theorem 6: Let the conditions of Theorem 2 hold, and

- $V: \Phi \longrightarrow \Phi$ be a bounded operator

- $\left(g\left(L_{2}\right)-1\right)^{-1} K \quad$ be a bounded operator in $\Phi$

then

$$
(g(\lambda)-1) y(\lambda) \stackrel{\Phi^{\prime}}{\longrightarrow} 0 \Rightarrow K^{\prime} y(\lambda) \rightarrow 0 \quad \text { as } \quad \lambda \rightarrow \infty .
$$

Proof:

$$
\begin{aligned}
<f, K^{\prime} y(\lambda)>_{\boldsymbol{\Phi} \times \Phi^{\prime}} & =<K f, y(\lambda)>_{\Phi \times \Phi^{\prime}} \\
& =\widehat{K f}^{2}(\lambda) \\
& =(g(\lambda)-1)(g(\lambda)-1)^{-1} \widehat{K f}^{2}(\lambda) \\
& =(g(\lambda)-1)\left\{\left(g\left(L_{2}\right)-1\right)^{-1} K f^{2}\right\} \\
& \left.=(g(\lambda)-1)<\left(g\left(L_{2}\right)-1\right)^{-1} K f\right), y(\lambda)>_{\Phi \times \Phi^{\prime}} \\
& \left.=<\left(g\left(L_{2}\right)-1\right)^{-1} K f\right),(g(\lambda)-1) y(\lambda)>_{\Phi \times \Phi^{\prime}}
\end{aligned}
$$

Since the $[g(\lambda)-1] y(\lambda) \stackrel{\Phi^{\prime}}{\rightarrow} 0$ we obtain $<f, K^{\prime} y(\lambda)>_{\Phi \times \Phi^{\prime}} \rightarrow 0 \quad \forall f \in \Phi$ and so $K^{\prime} y(\lambda) \rightarrow$ 0 as $\lambda \rightarrow \infty$.

Corollary 3: Assume that conditions of Theorem 4, hold and

- $y(\lambda)$ are bounded functionals for large $\lambda$

- $\left(g\left(L_{2}\right)-1\right)^{-1} K$ be a bounded operator in $\Phi$ 
then

$$
g(\lambda)-1 \stackrel{\lambda \rightarrow \infty}{\longrightarrow} 0 \Rightarrow y(\lambda)-\varphi(\lambda) \stackrel{\Phi^{\prime}}{\longrightarrow} 0 \text { as } \quad \lambda \rightarrow \infty
$$

Proof: It suffices to see that $(g(\lambda)-1) y(\lambda) \stackrel{\Phi^{\prime}}{\longrightarrow} 0$, and since Theorem 6, is applicable

$$
K^{\prime} y(\lambda) \rightarrow 0 \text { as } \lambda \rightarrow \infty .
$$

From Theorem 4, we deduce that

$$
g(\lambda) \varphi(\lambda)-y(\lambda) \stackrel{\Phi^{\prime}}{\longrightarrow} 0 .
$$

It remains to see that since $g(\lambda) \asymp 1$ as $\lambda \rightarrow \infty \Rightarrow \varphi(\lambda) \stackrel{\Phi^{\prime}}{\longrightarrow} y(\lambda)$ as $\lambda \rightarrow \infty$.

\section{EXAMPLES}

Below we shall consider two simple examples to illustrate the above results.

Let $L_{1}$ and $L_{2}$ be two self-adjoint differential operators in $L^{2}[0, \infty)$ defined by

$$
\left\{\begin{array} { l } 
{ L _ { 1 } f \equiv - f ^ { \prime \prime } ( x ) + q ( x ) f ( x ) } \\
{ n f ( 0 ) - f ^ { \prime } ( 0 ) = 0 }
\end{array} \text { and } \quad \left\{\begin{array}{l}
L_{2} f \equiv-f^{\prime \prime}(x) \\
n f(0)-f^{\prime}(0)=0
\end{array}\right.\right.
$$

where $|n|<\infty$. Let the eigenfunctionals associated with $L_{1}$ and $L_{2}$ be defined by

$$
\left\{\begin{array} { l } 
{ L _ { 1 } \varphi ( x , \lambda ) \equiv \lambda \varphi ( x , \lambda ) } \\
{ \varphi ( 0 , \lambda ) = 1 , \quad \varphi ^ { \prime } ( 0 , \lambda ) = n }
\end{array} \text { and } \left\{\begin{array}{l}
L_{2} y(x, \lambda)=\lambda y(x, \lambda) \\
y(0, \lambda)=1, y^{\prime}(0, \lambda)=n
\end{array}\right.\right.
$$

where $y(x, \lambda)=\cos (\sqrt{\lambda} x)+n \frac{\sin (\sqrt{\lambda} x)}{\sqrt{\lambda}}$. It is clear that

$$
\varphi(x, \lambda)=y(x, \lambda)+\int_{0}^{x} \frac{\sin (\sqrt{\lambda}(x-t))}{\sqrt{\lambda}} q(t) \varphi(t, \lambda) d t .
$$

By the Riemman-Lebesgue theorem we have

$$
\varphi(x, \lambda)-y(x, \lambda) \longrightarrow 0 \text { as } \lambda \longrightarrow \infty .
$$

It is also known that the following representation holds

$$
\varphi(x, \lambda)=y(x, \lambda)+\int_{0}^{x} K(x, t) y(t, \lambda) d t .
$$

Then formally

$$
\left(V^{\prime}-1\right) y(x, \lambda)=\int_{x}^{\infty} K(t, x) y(t, \lambda) d t
$$

Therefore if $\left(V^{\prime}-1\right) y(x, \lambda) \stackrel{\Phi^{\prime}}{\longrightarrow} 0$ then

$$
\frac{d \Gamma_{1}(\lambda)}{d \lambda} \asymp \frac{1}{\pi} \frac{\sqrt{\lambda}}{\lambda+n^{2}} \quad \text { as } \quad \lambda \rightarrow \infty
$$

Remark: It is known that if $q^{\prime}(x) \in L^{1, \text { loc }}[0, \infty)$ then for each fixed $x K_{t t}(x, t) \in L^{1, l o c}[0, \infty)$ and hence $L_{2} K$ is densely defined. Therefore Theorem 5 is applicable.

The next example deals with the generalized Sturm Liouville operator. Let

$\left\{\begin{array}{l}L_{1} f \equiv-\frac{1}{w(x)} f^{\prime \prime}(x)+q(x) f(x) \\ f^{\prime}(0)=0 .\end{array}\right.$ and $\left\{\begin{array}{l}L_{2} f \equiv \frac{-1}{x^{\alpha}} f^{\prime}(x) \\ f^{\prime}(0)=0 .\end{array}\right.$

where $w(x) \asymp x^{\alpha}$ as $x \rightarrow 0$ and $\alpha>0$. In this case the operator $L_{2}$ corresponds to a string whose length and mass are infinite, and is known to be self-adjoint in the space $L_{x^{\alpha} d x}^{2}$, see [5, p. 151] and [9]. 
We shall see that the behaviour of $w(x) \rightarrow 0$ dictates the behaviour of the spectral function at infinity. Although this result is known, see [6], we shall provide a different treatment as it is stated in [7]. For simplicity let the eigenfunctionals associated with $L_{1}$ and $L_{2}$ be defined by

$\left\{\begin{array}{l}L_{1} \varphi(x, \lambda) \equiv \lambda \varphi(x, \lambda) \\ \varphi(0, \lambda)=1, \varphi^{\prime}(0, \lambda)=0\end{array}\right.$ and $\quad\left\{\begin{array}{l}L_{2} y(x, \lambda)=\lambda y(x, \lambda) \\ y(0, \lambda)=1, y^{\prime}(0, \lambda)=0 .\end{array}\right.$

It is clear that

$$
\varphi(x, \lambda)=y(x, \lambda)+\int_{0}^{x} R(x, t, \lambda) q(t) \varphi(t, \lambda) d t .
$$

where $R(x, t, \lambda)$ is the Greens' function and it is shown, by the semi-classical approximation, see [8], that $R(x, t, \lambda) \longrightarrow 0$ as $\lambda \rightarrow \infty$. Therefore we have that $\varphi(x, \lambda)-y(x, \lambda) \longrightarrow 0$ as $\lambda \rightarrow \infty$. The solution $y(x, \lambda)$ are known explicitly,

$$
y(x, \lambda)=\sqrt{x} A J_{-\nu}\left(\left(\frac{2 \sqrt{\lambda}}{\alpha+2}\right) x^{\frac{a+2}{2}}\right) .
$$

where $\nu=\frac{1}{\alpha+2}$ and $A=\left\{\frac{2 \sqrt{\lambda}}{\alpha+2}\right\}^{\frac{1}{\alpha+2}} \frac{1}{\Gamma(1-\nu)}$.

Therefore provided $\left(V^{\prime}-1\right) y(x, \lambda) \stackrel{\Phi^{\prime}}{\longrightarrow} 0$, we shall have

$$
\Gamma_{1}(\lambda) \asymp \Gamma_{2}(\lambda) \text { as } \lambda \rightarrow \infty .
$$

where, see [3], $\Gamma_{2}(\lambda)=c \lambda^{\frac{\alpha+1}{\alpha+2}}$ for $\lambda>0$.

Acknowledgment: It is a pleasure to acknowledge the support of K.F.U.P.M. while the author was visiting the University of Illinois, at Urbana-Champaign.

\section{REFERENCES}

[1] BEREZANSKI, Ju.M., Expansions in Eigenfunctions of Selfadjoint Operators, Amer. Math. Transl. Mono, Vol. 17, 1968.

[2] GELFAND, I.M. and SHILOV, G. Generalized Functions, Academic Press, 1963.

[3] BOUMENIR, A., A comparison theorem for self-adjoint operators, Proc. Amer. Math. Soc, 11, 1 (1991), 161-175.

[4] DIEUdonneE, J., Treatise in Analysis, Vol. 2, Academic Press, 1976.

[5] DYM, H. and McKEAN, H.P., Gaussian Processes, Function Theoritic and the Inverse Spectral Problem, Academic Press, 1976

[6] BENNEWITZ, C.S., Spectral asymptotics for Sturm-Liouville equations, Proc. London. Math. Soc. 3, 59 (1989) 294-338.

[7] KAC, I.S., Power-Asymptotic estimates for spectral functions of generalized boundary value problems of second order, Sov. Math. Dokl., Vol. 13 (1972), No. 2, 453-455.

[8] NIKIFOROV, A. and OUVAROV, V., Fonctions speciales de la physique, Mathematique, M.I.R Publishers, 1983, p. 178.

[9] KREIN, M.G. and KAC, I.S., Spectral function of the string, Amer. Math. Soc. Transl., 2, Vol. 103 (1970), 19-103. 


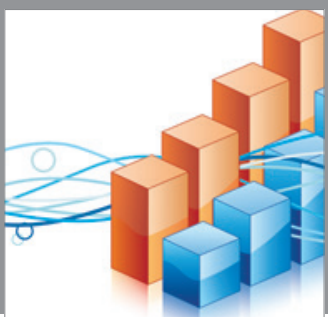

Advances in

Operations Research

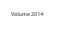

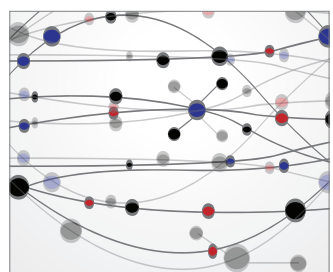

\section{The Scientific} World Journal
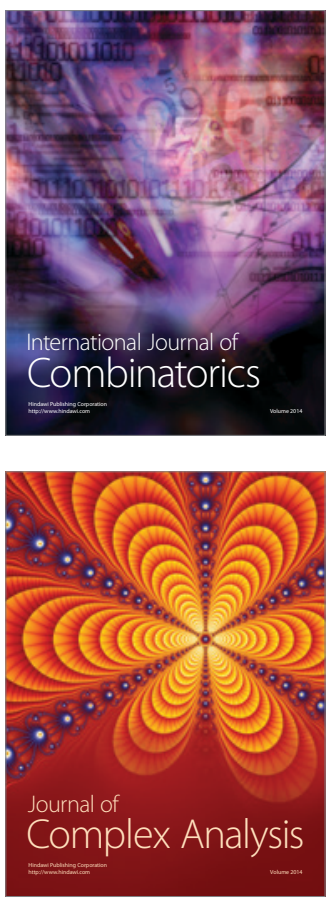

International Journal of

Mathematics and

Mathematical

Sciences
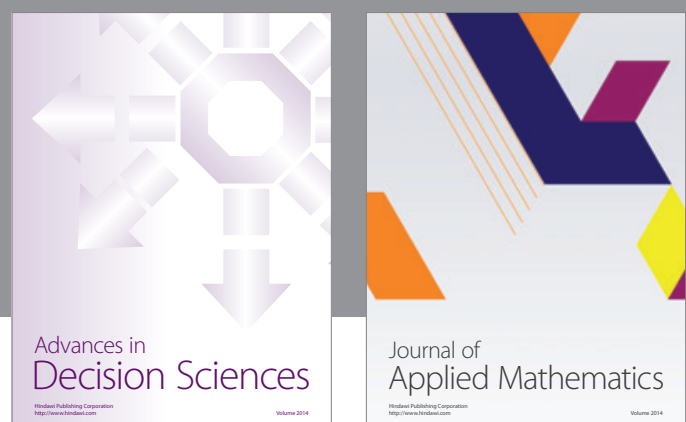

Journal of

Applied Mathematics
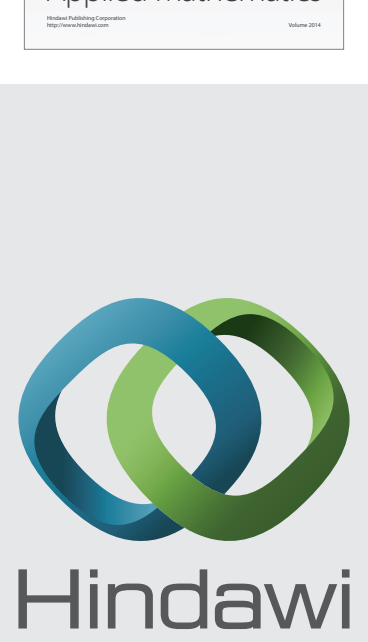

Submit your manuscripts at http://www.hindawi.com
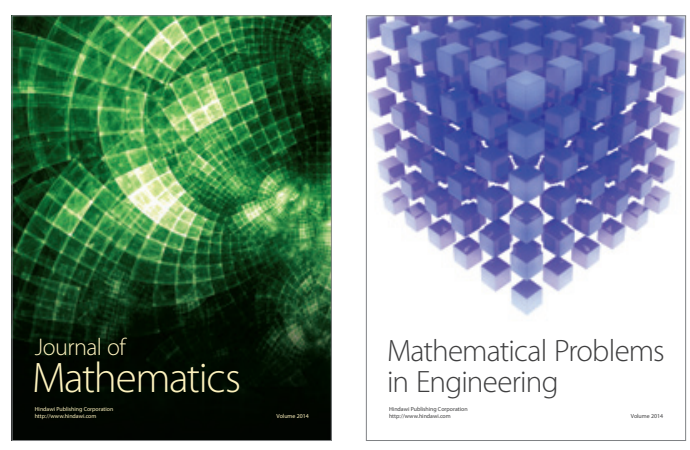

Mathematical Problems in Engineering
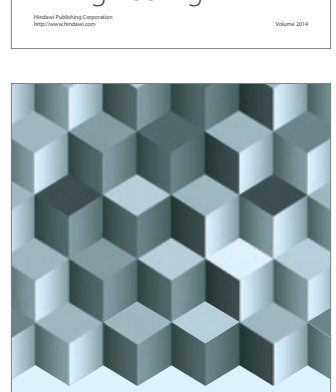

Journal of

Function Spaces
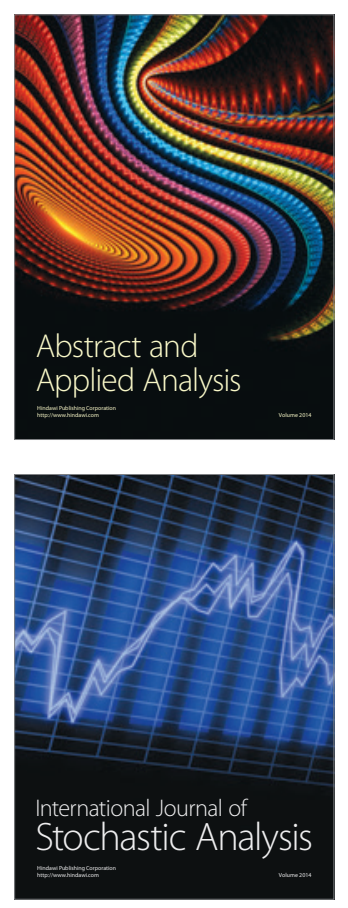

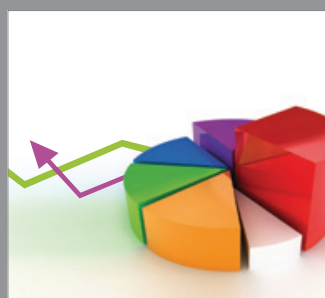

ournal of

Probability and Statistics

Promensencen
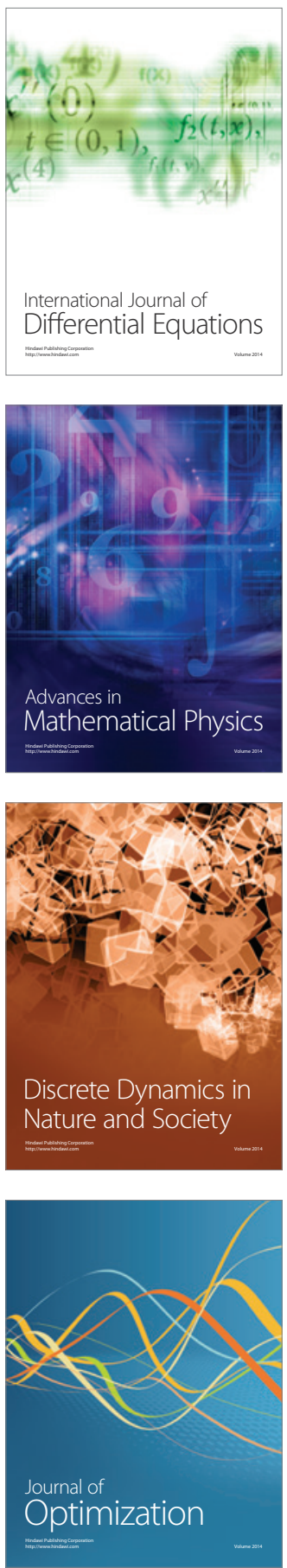